\title{
La metabolomica nella diagnosi delle patologie da ipersecrezione e dei tumori corticali surrenalici
}

\author{
Guido Di Dalmazi ${ }^{1,2}$
}

Accettato: 26 gennaio 2021 / Pubblicato online: 14 settembre 2021

(c) The Author(s) 2021

\begin{abstract}
Sommario La metabolomica ha permesso un notevole avanzamento nella comprensione della fisiopatologia delle ghiandole surrenaliche. L'applicazione della spettrometria di massa alla misurazione dei metaboliti ha portato all' $\mathrm{i}-$ dentificazione di pannelli con enormi potenzialità in ambito diagnostico, come ad esempio nell'identificazione della patologia surrenalica espansiva maligna, nella predizione delle recidive e nella diagnosi di sottotipo dell'ipersecrezione glucocorticoide e mineralcorticoide.
\end{abstract}

Parole chiave Metabolomica $\cdot$ Steroidi $\cdot$ Spettrometria di massa . Tumori surrenalici - Ipercortisolismo .

Iperaldosteronismo

\section{Introduzione}

La metabolomica, ossia lo studio dei processi metabolici mediante misurazione di metaboliti di piccole dimensioni $(<1500 \mathrm{Da})$, è un campo in rapida espansione [1]. Si colloca al termine della cosiddetta cascata -omica, che include lo studio di processi biologici, quali meccanismi regolatori dei geni (genomica) e dei loro prodotti (trascrittomica e proteomica). Grazie alla capacità intrinseca di fornire un quadro di insieme di tutti i processi metabolici che avvengono

Proposto da Carla Scaroni.

Informazioni Supplementari La versione online contiene materiale supplementare disponibile su

https://doi.org/10.1007/s40619-021-00957-7.

$凶$ G. Di Dalmazi

guido.didalmazi@unibo.it

1 UOC di Endocrinologia e Prevenzione e Cura del Diabete, IRCCS Azienda Ospedaliero-Universitaria di Bologna, Bologna, Italia

2 Dipartimento di Scienze Mediche e Chirurgiche, Università di Bologna, Bologna, Italia in un determinato campione biologico in un preciso istante, l'applicazione della metabolomica allo studio delle patologie surrenaliche ha permesso dei notevoli avanzamenti nella comprensione della fisiopatologia dei tumori e dell'ipersecrezione ormonale. In base all'approccio analitico, la metabolomica può essere classificata in untargeted (analisi di pannelli molto ampi, non mirati all'identificazione di metaboliti noti a priori) o targeted, ossia mirata allo studio di metaboliti specifici, noti e definiti a priori. Nell'ambito di quest'ultimo approccio, l'analisi dei profili steroidei (steroidometabolomica) riveste un ruolo di primaria importanza per lo studio delle patologie surrenaliche.

In questa rassegna verranno presentate le principali applicazioni della metabolomica alla diagnosi dei tumori surrenalici, con particolare attenzione ai dati più recenti nella letteratura.

\section{Brevi cenni sulle metodiche di analisi metabolomica}

Le tecniche di metabolomica si avvalgono di misurazioni che richiedono una specificità elevata. In questo campo, la spettrometria di massa (MS) svolge un ruolo di rilievo assoluto. I parametri su cui si basa la MS sono il peso e la struttura delle molecole. I processi analitici basilari sono ionizzazione, separazione e rilevazione. Fra le principali metodiche di separazione cromatografica, particolarmente rilevanti per l'analisi degli steroidi per via della loro similarità strutturale, vi sono la gas-cromatografia (GC) e la cromatografia liquida (LC).

La GC-MS presenta un elevato potere di risoluzione e ha applicazioni in diversi ambiti (industria alimentare, tossicologia, medicina legale e farmacologia). Nella ricerca medica e, in particolare, nello studio delle patologie surrenaliche, 
la GC-MS è stata applicata alla metabolomica untargeted e alla steroido-metabolomica su urine delle 24 ore. Tuttavia, l'alta risoluzione della GC-MS si accompagna a una laboriosa preparazione del campione, che rende questa metodica poco applicabile alla pratica routinaria.

La LC-MS è invece di più rapida esecuzione e ad alto volume di produzione, sebbene con una capacità di risoluzione inferiore alla GC-MS. Questa tecnica è maggiormente utile per piccoli pannelli, fino a 20 metaboliti, ed è trasferibile in pratica clinica. Nello studio delle patologie surrenaliche, la LC-MS è stata largamente utilizzata per l'analisi dei profili steroidei. A tale scopo vengono tipicamente utilizzati uno spettrometro di massa tandem (MS/MS) costituito da due quadrupoli a trasmissione ionica posizionati su una cella di collisione. Il primo quadrupolo seleziona uno specifico ione rappresentativo del metabolita, che viene frazionato nella cella di collisione in ioni specifici, ulteriormente selezionati dal secondo quadrupolo, che incrementa la sensibilità della metodica, e trasmessi al rilevatore.

In entrambe le metodiche viene, infine, generato un cromatogramma, dove l' area del picco è proporzionale alla concentrazione del metabolita, la cui quantificazione avviene mediante confronto con materiale di riferimento.

\section{Metabolomica untargeted}

Vi sono pochi studi sulla metabolomica untargeted nelle patologie surrenaliche, a causa di elevati costi, tempo e complessità delle analisi. Questi studi sono principalmente focalizzati sulla distinzione della patologia surrenalica maligna da quella benigna. In un elegante studio del 2013 su 66 tessuti surrenalici, i profili di metabolomica untargeted mediante spettroscopia di risonanza magnetica nucleare hanno differenziato i tessuti tumorali da quelli normali di medesima derivazione embriologica. Inoltre, i carcinomi surrenalici mostravano un profilo specifico caratterizzato da biomarcatori di processi anaerobici, attività glicolitica e metabolismo degli aminoacidi, nettamente distinto dai tumori benigni e dai tessuti normali. Nonostante la precisione diagnostica di questo approccio, l'accuratezza nel distinguere la malattia metastatica da quella non metastatica non è risultata soddisfacente [2]. Recentemente, l'utilizzo di profili metabolomici su un singolo campione di urine delle 24 ore ha mostrato un' accuratezza dell' $84 \%$ nel classificare correttamente i carcinomi surrenalici, con un'area sotto la curva (AUC) di 0,93. Tra i 46 metaboliti più significativi, la creatina riboside (elevato anche in tumori del polmone) e 4 metaboliti non identificati hanno mostrato una buona accuratezza diagnostica anche in un set indipendente di validazione. La combinazione di livelli di creatina riboside e dimensione della massa surrenalica ha mostrato sensibilità dell' $80 \%$, specificità del $96 \%$, valore predittivo negativo
(VPN) del 98\%, ma con un valore predittivo positivo (VPP) insoddisfacente (57\%) [3].

\section{Metabolomica targeted}

\section{Metabolismo intermedio}

Recentemente, i profili metabolomici di pazienti con ipercortisolismo sono stati analizzati con LC-MS/MS. La misurazione di un pannello di 180 metaboliti plasmatici (aminoacidi, amine biogeniche, acilcarnitine e lipidi) ha permesso una corretta classificazione dei 149 soggetti nei 4 gruppi in studio (controlli, incidentalomi surrenalici non secernenti, ipercortisolismo subclinico e sindrome di Cushing) con sensibilità, specificità, VPP e VPN maggiori di $80 \%$. Inoltre, sono stati evidenziati 4 distinti profili metabolomici in base alla presenza o meno di ipercortisolismo e diabete. L'analisi dei singoli metaboliti ha identificato alterazioni del metabolismo proteico (riduzione di aminoacidi a catena ramificata, istidina, triptofano e kinurenina, e incremento delle poliamine) e glucidico (riduzione di carnitina e acetil-carnitina e incremento di acilcarnitine a lunga catena), compatibili con inflessibilità metabolica [4].

\section{Profili steroidei}

I dati principali degli studi di steroido-metabolomica sono riassunti nelle Tabelle 1 e 2.

\section{Carcinoma surrenalico}

Uno dei primi studi che ha mostrato la rilevanza clinica dei profili steroidei nella diagnosi di carcinoma surrenalico è stato pubblicato nel 2011 e riportava i risultati di un lavoro collaborativo dello European Network for the Study of Adrenal Tumors (ENSAT). L'analisi di un largo pannello di steroidi, eseguita mediante GC-MS su urine delle 24 ore di 147 pazienti, ha permesso di identificare un profilo secretorio specifico del carcinoma surrenalico e un pannello di 9 steroidi selezionati, che avevano un'accuratezza diagnostica soddisfacente nell'identificare i carcinomi surrenalici [5]. I precursori glucocorticoidi, come il tetraidro-11-desossicortisolo (THS), metabolita urinario dell'11-desossicortisolo, e degli ormoni sessuali, come il pregnanediolo (5-PD) e il pregnanetriolo (5-PT), principali metaboliti urinari del progesterone e del 17idrossiprogesterone, rispettivamente, erano fra i principali metaboliti discriminanti [5]. Tali dati sono stati confermati in tre studi indipendenti, che hanno utilizzato la GC-MS e la LC-MS/MS per l'analisi del profilo steroideo su urine delle 24 ore. Il primo, su 152 pazienti, ha evidenziato il potenziale ruolo diagnostico di THS, 5-PD e 5-PT fra i 7 metaboliti 


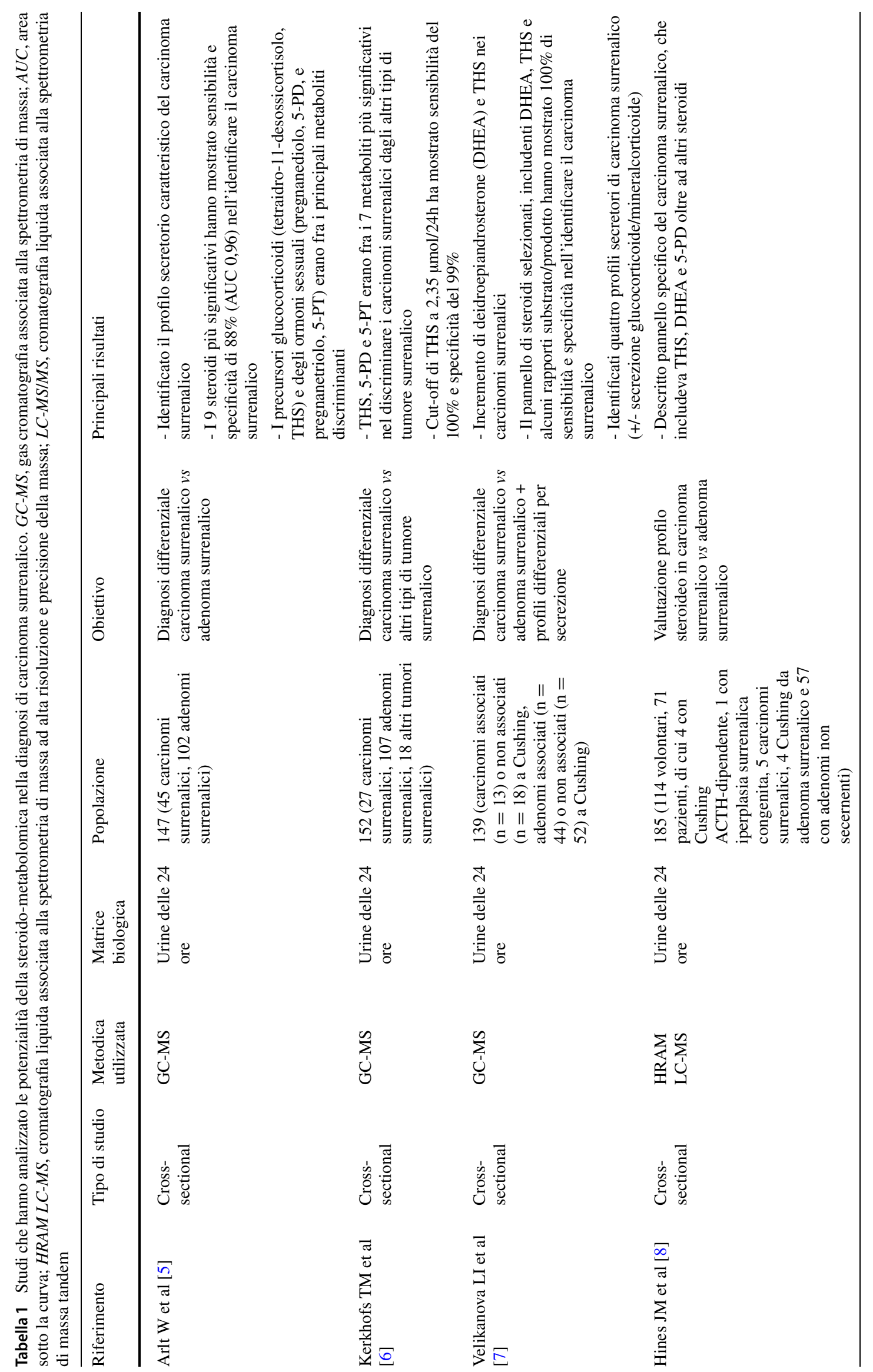




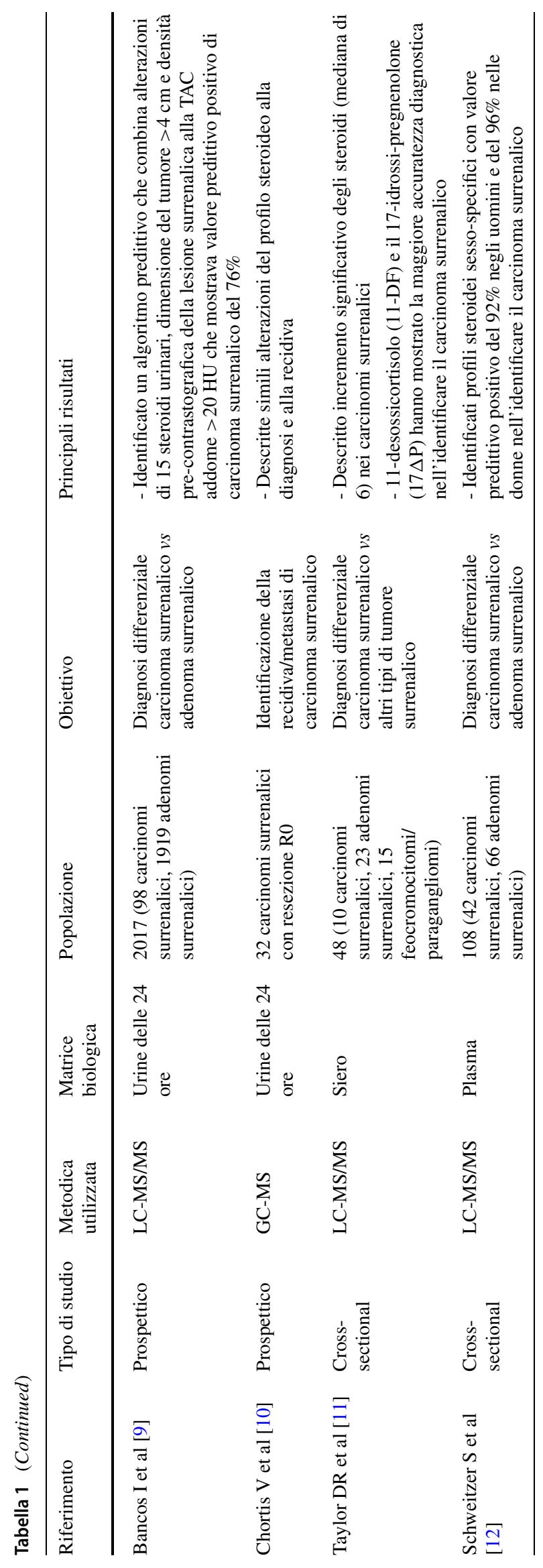




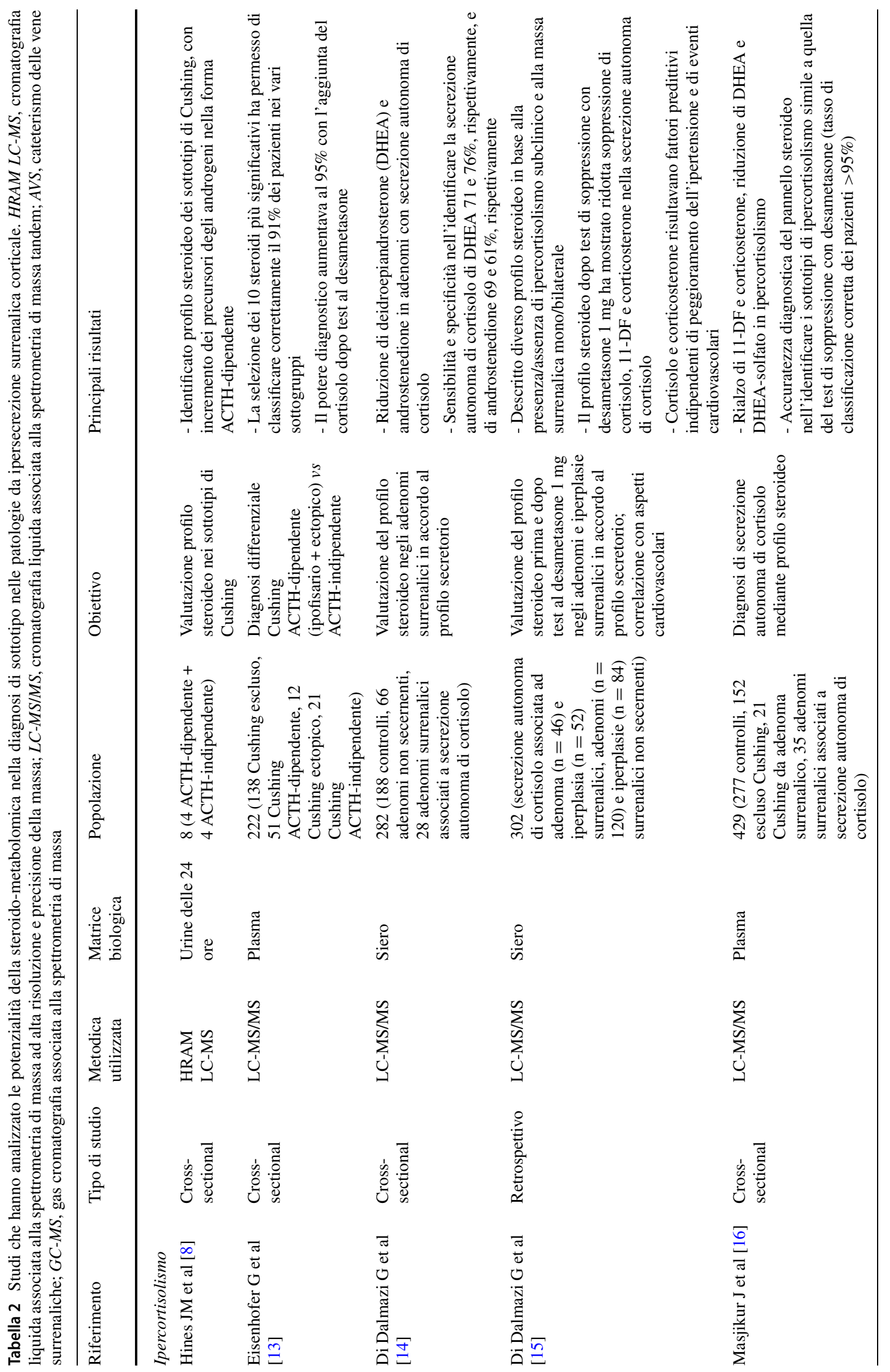




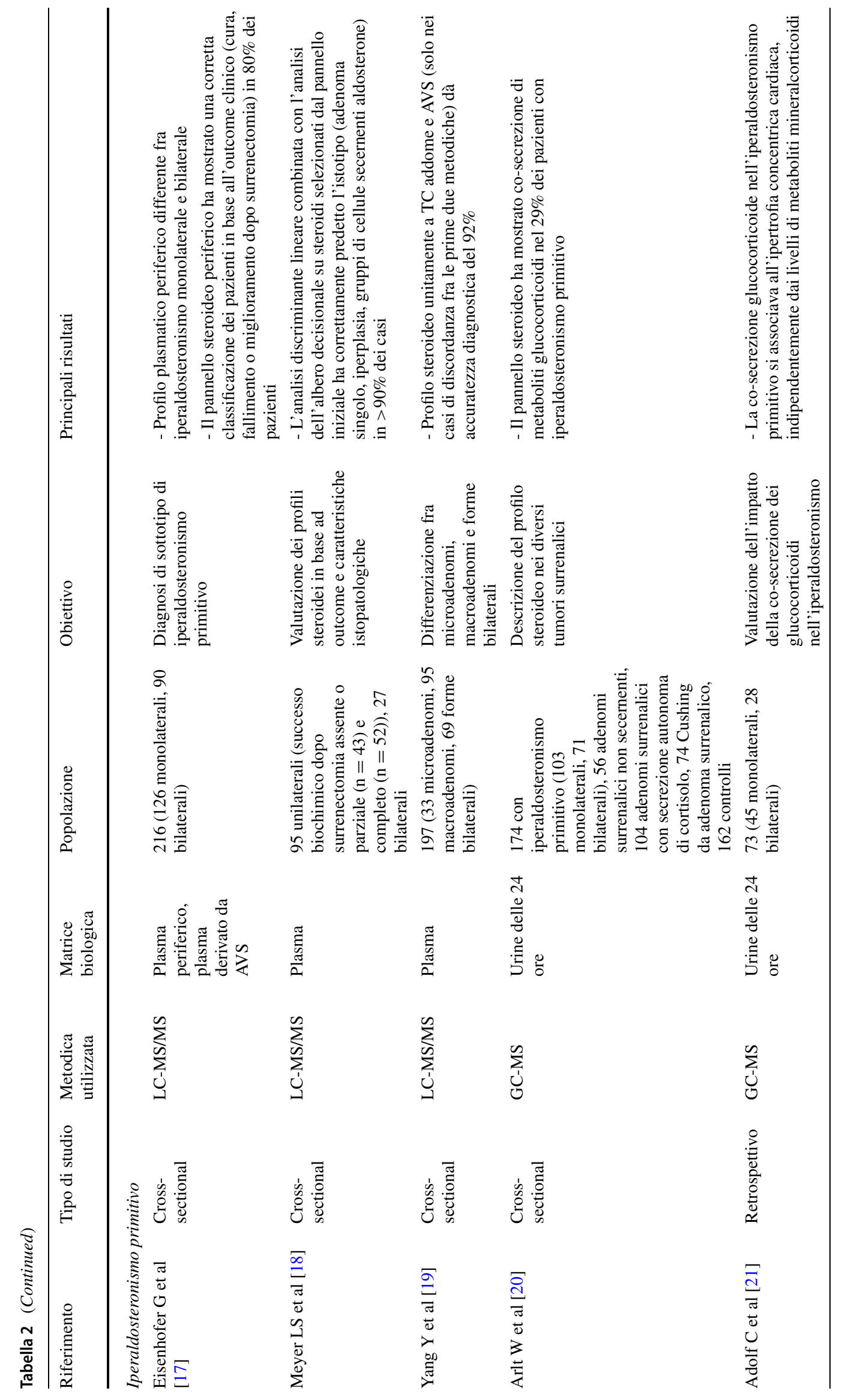


più significativi nel distinguere i carcinomi surrenalici dagli altri tipi di tumore surrenalico, con una sensibilità del $100 \%$ [6]. Il secondo, oltre a confermare la potenzialità diagnostica del profilo steroideo urinario nel differenziare il carcinoma dall'adenoma surrenalico, ha mostrato l'esistenza di 4 diversi profili secretori di carcinoma surrenalico, in base alla presenza o meno di secrezione glucocorticoide e mineralcorticoide [7]. Il terzo studio ha sia confermato le potenzialità diagnostiche del profilo steroideo, sia rimarcato il ruolo primario del rialzo di THS e dei metaboliti dei precursori androgenici nell'identificazione del carcinoma surrenalico [8]. Recentemente, i dati sul profilo steroideo urinario sono stati validati prospetticamente mediante LC-MS/MS in uno studio ENSAT, che ha incluso 2017 pazienti con tumori surrenalici, di cui 98 con carcinoma surrenalico. L'analisi del profilo steroideo urinario, ristretta ai 15 metaboliti più significativi secondo il primo studio del 2011, è stata eseguita mediante LC-MS/MS. I risultati dello studio hanno messo in evidenza che la combinazione di alterazioni del pannello di 15 steroidi urinari indicativi di alto rischio di malignità e di dati radiologici (dimensione del tumore $>4 \mathrm{~cm}$ e densità pre-contrastografica della lesione surrenalica alla TAC addome $>20 \mathrm{HU}$ ) avevano un valore predittivo positivo di carcinoma surrenalico del 76\% [9].

I dati sul potenziale diagnostico del profilo steroideo urinario sono stati estesi in un recente studio pilota, che ha analizzato l'utilità della steroidomica nell'identificare le recidive di carcinoma surrenalico, per superare le attuali limitazioni delle tecniche di imaging. Il profilo steroideo è stato eseguito su urine delle 24 ore raccolte a cadenza trimestrale in pazienti con carcinoma surrenalico con resezione R0, con follow-up mediano di 32 mesi. Nei $13 / 32$ pazienti con recidiva, le stesse alterazioni dei metaboliti steroidei rilevate nel preoperatorio sono state identificate alla comparsa della recidiva. Tuttavia, la sensibilità nell'identificare la recidiva è risultata insoddisfacente (56\%) [10].

L'utilizzo dei profili steroidei nella diagnosi di carcinoma surrenalico è stato anche esteso ai derivati ematici (plasma e siero), mediante LC-MS/MS. Il primo studio ha analizzato un pannello di 13 steroidi sierici di 10 pazienti con carcinoma surrenalico e 38 pazienti con lesioni surrenaliche non carcinomatose, evidenziando un rialzo significativo degli steroidi (mediana di 6) nel primo gruppo. L'11desossicortisolo e il 17-idrossi-pregnenolone risultavano fra gli steroidi con maggiore accuratezza diagnostica, in linea con i risultati degli studi eseguiti sulle urine delle 24 ore [11]. Uno studio indipendente più recente, che ha analizzato un pannello di 15 steroidi plasmatici in 42 pazienti con carcinoma surrenalico e 66 con adenoma surrenalico, ha mostrato l'esistenza di profili steroidei sesso-specifici, composti da 6 steroidi ognuno, che distinguevano il carcinoma dall'adenoma surrenalico con un valore predittivo positivo del $92 \%$ negli uomini e del $96 \%$ nelle donne [12].

\section{Tumori surrenalici associati a ipercortisolismo}

Negli ultimi anni sono emersi studi che hanno valutato la potenzialità del profilo steroideo nell'eseguire la diagnosi di sottotipo nella sindrome di Cushing. Lo studio di Hines e collaboratori menzionato in precedenza ha mostrato dati pilota su 8 pazienti con sindrome di Cushing, sottolineando che il profilo secretorio steroideo su urine delle 24 ore si caratterizzava per la differente secrezione di metaboliti dei precursori androgenici, che risultavano elevati nel sottotipo ACTH-dipendente e ridotti in quello ACTH-indipendente [8]. In un successivo studio indipendente, mirato specificamente a questa analisi e su una casistica più ampia $(n=21$ sindrome di Cushing di origine surrenalica, $\mathrm{n}=51$ di origine ipofisaria e 12 ectopici), è stato invece analizzato un pannello di 15 steroidi plasmatici per eseguire la diagnosi di sottotipo. Lo studio ha confermato la presenza di diversi profili secretori dei precursori androgenici, ma ha anche rilevato un ruolo nei livelli di aldosterone e 18-oxo-cortisolo (uno steroide ibrido di derivazione dal 11-desossicortisolo) nel distinguere i 3 sottotipi di sindrome di Cushing. Inoltre, selezionando i 10 steroidi più significativi, il solo profilo steroideo plasmatico è stato in grado di classificare correttamente il $91 \%$ della popolazione in studio; l'aggiunta dei valori di cortisolo dopo test al desametasone ha aumentato il potere diagnostico al $95 \%$ [13].

Lo studio del profilo steroideo circolante è stato esteso anche alle forme di ipercortisolismo subclinico. L'analisi di un pannello di 10 steroidi sierici eseguita su 94 pazienti con incidentaloma surrenalico e 188 controlli ha mostrato una riduzione, indipendente dall'età, di androgeni e precursori, principalmente deidroepiandrosterone (DHEA) e androstenedione, in adenomi con ipercortisolismo subclinico vs adenomi non secernenti. DHEA e androstenedione, singolarmente, hanno mostrato una sensibilità di $71 \mathrm{e}$ $69 \%$, rispettivamente, e una specificità di 76 e $61 \%$, rispettivamente, nell'identificare l'ipercortisolismo subclinico [14]. Tali risultati sono stati ampliati in un successivo studio, in cui il pannello di steroidi sierici è stato analizzato su 302 pazienti con incidentaloma surrenalico, mettendo in evidenza un diverso profilo steroideo in base alla presenza/assenza di ipercortisolismo subclinico e alla massa surrenalica mono/bilaterale. Inoltre, i profili steroidei misurati dopo test di soppressione con desametasone $1 \mathrm{mg}$ hanno mostrato, nei pazienti con ipercortisolismo subclinico, una ridotta soppressione non solo di cortisolo, ma anche di 11desossicortisolo e corticosterone. Infine, i livelli di cortisolo e corticosterone si sono dimostrati fattori predittivi indipendenti di peggioramento dell'ipertensione e di eventi cardiovascolari [15]. Un ulteriore studio indipendente su 208 pazienti ha mostrato risultati in linea con i precedenti, confermando il rialzo di 11-desossicortisolo e corticosterone, e bassi livelli di DHEA e DHEAs nel plasma di pazienti con 
ipercortisolismo sia subclinico sia conclamato, se confrontati con soggetti di controllo. Il pannello di soli 14 steroidi ha mostrato un' accuratezza diagnostica simile a quella del test di soppressione con desametasone, con AUC di 0,96 e 0,94, rispettivamente e un tasso di classificazione corretta dei pazienti $>95 \%$ (97\% nel caso di ipercortisolismo subclinico) [16].

\section{Tumori surrenalici associati a iperaldosteronismo}

Dopo i primi studi pionieristici degli anni ' 80 che hanno mostrato un rialzo di steroidi ibridi in alcune forme di iperaldosteronismo, il ruolo di 18-oxo-cortisolo (18-oxo-F) e 18idrossi-cortisolo (18-OH-F), prodotti dall'aldosterone sintasi a partire dall'11-desossicortisolo e sotto prevalente controllo dell' ACTH, è diventato di crescente interesse. In uno studio del 2016 è stato valutato un pannello di 15 steroidi plasmatici che includeva anche gli steroidi ibridi, misurati da sangue periferico e da cateterismo delle vene surrenaliche (AVS) mediante LC-MS/MS, in 216 pazienti con iperaldosteronismo primitivo. Il profilo plasmatico periferico è risultato differente fra iperaldosteronismo monolaterale e bilaterale, con un rialzo del 18-oxo-F nel primo. Sebbene questo steroide, singolarmente, non abbia mostrato soddisfacente specificità e sensibilità nel distinguere le forme mono da quelle bilaterali, il potere diagnostico cresceva in maniera significativa considerando il pannello di steroidi nel suo insieme (AUC $=0,89$ ). L'utilizzo dei 15 steroidi plasmatici periferici ha anche permesso una corretta classificazione dei pazienti in base all'esito della surrenectomia (cura, miglioramento o fallimento) in 172/216 pazienti (80\%) [17]. Questi risultati sono stati estesi in uno studio che ha analizzato i profili steroidei plasmatici periferici in differenti istotipi di iperaldosteronismo primitivo (adenoma singolo, iperplasia e gruppi di cellule secernenti aldosterone - aldosterone producing cell clusters, APCC). Sebbene non fossero state rilevate differenze significative nelle concentrazioni di steroidi circolanti fra le tre condizioni istopatologiche, l'analisi discriminante lineare combinata con l'analisi dell' albero decisionale su steroidi selezionati ha permesso di eseguire una corretta predizione dell' istotipo in più del $90 \%$ dei casi [18].

Un ulteriore studio ha analizzato il profilo steroideo periferico in 197 pazienti con iperaldosteronismo primitivo, mostrando, nella maggior parte dei casi, risultati concordanti con la TAC nel classificare correttamente i pazienti affetti da malattia monolaterale da micro- e macro-adenoma $(<\mathrm{e}$ $>10 \mathrm{~mm}$ di diametro, rispettivamente), e da malattia bilaterale. La classificazione ottenuta mediante la combinazione del profilo steroideo e della TAC addome ha mostrato risultati concordanti, anche in termini di outcome clinico e biochimico, con quelli ottenuti mediante AVS in 163/197 casi (83\%). Pertanto, in accordo a questo studio, l'algoritmo decisionale dei pazienti con iperaldosteronismo primitivo potrebbe essere implementato dalla combinazione del pannello di steroidi e della TAC addome, che permetterebbe di ridurre il numero di AVS a una piccola percentuale di pazienti [19]. L'applicazione della misurazione del pannello steroideo in GC-MS su urine delle 24 ore in pazienti con iperaldosteronismo primitivo ha permesso di identificare la presenza di cosecrezione di metaboliti glucocorticoidi in una percentuale non trascurabile di pazienti (29\%) [20]. In uno studio indipendente, la co-secrezione glucocorticoide nell'iperaldosteronismo primitivo, identificata mediante medesima metodica utilizzata nel precedente studio, si associava alla presenza di ipertrofia cardiaca concentrica, indipendentemente dai livelli di mineralcorticoidi [21].

\section{Direzioni future}

Le tecniche di metabolomica hanno permesso di approfondire la fisiopatologia dei tumori surrenalici e di pianificare strategie diagnostiche che saranno di comune uso nella pratica clinica nel corso dei prossimi anni. La visione futura offerta dalla metabolomica prevede una semplificazione diagnostica delle patologie da ipersecrezione ormonale e una più accurata identificazione delle lesioni maligne surrenaliche, all'esordio e alla recidiva. Inoltre, in un'ottica di valutazione multi-analitica, la combinazione di metabolomica e di altre tecniche di -omica potrà fornire nozioni di fisiopatologia dei tumori surrenalici finora inesplorate, in cui elementi di anatomia patologica, biochimica molecolare e fisiologia vengono combinati insieme sotto l'egida, ineludibile, del supporto informatico.

Questo avanzamento, tuttavia, avviene a un costo economico non indifferente, in termini di macchinari, personale e intelligenza artificiale, che limita la diffusione di queste metodiche a pochi centri specializzati. Infine, il miglioramento tecnologico richiede una crescente familiarità con algoritmi predittivi e modelli matematici, che dovranno essere integrati necessariamente alla raffinatezza della valutazione clinica, che rimane, comunque, una prerogativa centrale dell'endocrinologo.

Funding Note Open access funding provided by Alma Mater Studiorum - Università di Bologna within the CRUI-CARE Agreement.

Conflitto di interesse L'autore Guido Di Dalmazi dichiara di non avere conflitti di interesse.

Consenso informato Lo studio presentato in questo articolo non ha richiesto sperimentazione umana.

Studi sugli animali L'autore di questo studio non ha eseguito studi sugli animali.

Nota della casa editrice Springer Nature rimane neutrale in riguardo alle rivendicazioni giurisdizionali nelle mappe pubblicate e nelle affiliazioni istituzionali. 
Open Access This article is licensed under a Creative Commons Attribution 4.0 International License, which permits use, sharing, adaptation, distribution and reproduction in any medium or format, as long as you give appropriate credit to the original author(s) and the source, provide a link to the Creative Commons licence, and indicate if changes were made. The images or other third party material in this article are included in the article's Creative Commons licence, unless indicated otherwise in a credit line to the material. If material is not included in the article's Creative Commons licence and your intended use is not permitted by statutory regulation or exceeds the permitted use, you will need to obtain permission directly from the copyright holder. To view a copy of this licence, visit http://creativecommons.org/licenses/by/4.0/.

\section{Bibliografia}

1. Adamski J, Suhre K (2013) Metabolomics platforms for genome wide association studies-linking the genome to the metabolome. Curr Opin Biotechnol 24:39-47

2. Imperiale A, Elbayed K, Moussallieh FM et al (2013) Metabolomic profile of the adrenal gland: from physiology to pathological conditions. Endocr-Relat Cancer 20:705-716

3. Patel D, Thompson MD, Manna SK et al (2017) Unique and novel urinary metabolomic features in malignant versus benign adrenal neoplasms. Clin Cancer Res 23:5302-5310

4. Di Dalmazi G, Quinkler M, Deutschbein T et al (2017) Cortisolrelated metabolic alterations assessed by mass spectrometry assay in patients with Cushing's syndrome. Eur J Endocrinol 177:227-237

5. Arlt W, Biehl M, Taylor AE et al (2011) Urine steroid metabolomics as a biomarker tool for detecting malignancy in adrenal tumors. J Clin Endocrinol Metab 96:3775-3784

6. Kerkhofs TM, Kerstens MN, Kema IP et al (2015) Diagnostic value of urinary steroid profiling in the evaluation of adrenal tumors. Horm Cancer 6:168-175

7. Velikanova LI, Shafigullina ZR, Lisitsin AA et al (2016) Different types of urinary steroid profiling obtained by high-performance liquid chromatography and gas chromatography-mass spectrometry in patients with adrenocortical carcinoma. Horm Cancer 7:327-335

8. Hines JM, Bancos I, Bancos C et al (2017) High-Resolution, Accurate-Mass (HRAM) mass spectrometry urine steroid profiling in the diagnosis of adrenal disorders. Clin Chem 63:1824-1835
9. Bancos I, Taylor AE, Chortis V et al (2020) Urine steroid metabolomics for the differential diagnosis of adrenal incidentalomas in the EURINE-ACT study: a prospective test validation study. Lancet Diabetes Endocrinol 8:773-781

10. Chortis V, Bancos I, Nijman T et al (2020) Urine steroid metabolomics as a novel tool for detection of recurrent adrenocortical carcinoma. J Clin Endocrinol Metab 105:e307-e318

11. Taylor DR, Ghataore L, Couchman L et al (2017) A 13-steroid serum panel based on LC-MS/MS: use in detection of adrenocortical carcinoma. Clin Chem 63:1836-1846

12. Schweitzer S, Kunz M, Kurlbaum M et al (2019) Plasma steroid metabolome profiling for the diagnosis of adrenocortical carcinoma. Eur J Endocrinol 180:117-125

13. Eisenhofer G, Masjkur J, Peitzsch M et al (2018) Plasma steroid metabolome profiling for diagnosis and subtyping patients with Cushing syndrome. Clin Chem 64:586-596

14. Di Dalmazi G, Fanelli F, Mezzullo M et al (2015) Steroid profiling by LC-MS/MS in nonsecreting and subclinical cortisolsecreting adrenocortical adenomas. J Clin Endocrinol Metab 100:3529-3538

15. Di Dalmazi G, Fanelli F, Zavatta G et al (2020) The steroid profile of adrenal incidentalomas: subtyping subjects with high cardiovascular risk. J Clin Endocrinol Metab 104:5519-5528

16. Masjkur J, Gruber M, Peitzsch M et al (2019) Plasma steroid profiles in subclinical compared with overt adrenal Cushing syndrome. J Clin Endocrinol Metab 104:4331-4340

17. Eisenhofer G, Dekkers T, Peitzsch M et al (2016) Mass spectrometry-based adrenal and peripheral venous steroid profiling for subtyping primary aldosteronism. Clin Chem 62:514-524

18. Meyer LS, Wang X, Sušnik E et al (2018) Immunohistopathology and steroid profiles associated with biochemical outcomes after adrenalectomy for unilateral primary aldosteronism. Hypertension 72:650-657

19. Yang Y, Burrello J, Burrello A et al (2019) Classification of microadenomas in patients with primary aldosteronism by steroid profiling. J Steroid Biochem Mol Biol 189:274-282

20. Arlt W, Lang K, Sitch AJ et al (2017) Steroid metabolome analysis reveals prevalent glucocorticoid excess in primary aldosteronism. JCI Insight 2:e93136

21. Adolf C, Köhler A, Franke A et al (2018) Cortisol excess in patients with primary aldosteronism impacts left ventricular hypertrophy. J Clin Endocrinol Metab 103:4543-4552 